BMJ Open

Diabetes

Research

\& Care

\section{Association between liver enzymes and incident type 2 diabetes in Singapore Chinese men and women}

To cite: Wang Y-L, Koh W-P, Yuan J-M, et al. Association between liver enzymes and incident type 2 diabetes in Singapore Chinese men and women. BMJ Open Diabetes Research and Care 2016;4: e000296. doi:10.1136/ bmjdrc-2016-000296

- Additional material is available. To view please visit the journal (http://dx.doi.org/ 10.1136/bmjdrc-2016000296).

Received 8 July 2016 Revised 23 August 2016 Accepted 30 August 2016

\section{(1) CrossMark}

For numbered affiliations see end of article.

Correspondence to Professor An Pan; panan@hust.edu.cn

\section{ABSTRACT}

Aims: To assess the association between liver enzymes and the risk of type 2 diabetes (T2D) in a Chinese population.

Methods: A nested case-control study comprising 571 T2D cases and 571 matched controls was conducted within the Singapore Chinese Health Study. Alanine aminotransferase (ALT), aspartate aminotransferase (AST), alkaline phosphatase (ALP), and lactate dehydrogenase $(\mathrm{LDH})$ were quantified in baseline plasma collected from them, while $\gamma$ glutamyltransferase (GGT) was assayed among 255 T2D cases with baseline hemoglobin A1c $<6.5 \%$ and 255 matched controls. Participants were free of diagnosed diabetes, cardiovascular disease, and cancer at blood collections (1999-2004). Incident selfreported T2D cases were identified at follow-up II interview (2006-2010). Controls were matched to cases on age, sex, dialect group, and date of blood collection.

Results: Higher levels of ALT and GGT were significantly associated with increased risk of T2D ( $p$ for trend $<0.001$ for ALT, $p$ for trend=0.03 for GGT), and the $\mathrm{ORs}(95 \% \mathrm{Cls})$ comparing highest versus lowest tertiles of ALT and GGT were 2.00 (1.01 to 3.96 ) and 2.38 (1.21 to 4.66), respectively. A null association was observed for AST, ALP, and LDH with T2D risk. Adding GGT ( $<23$ vs $\geq 23 \mathrm{IU} / \mathrm{L}$ ) or ALT ( $<21 \mathrm{vs} \geq 21 \mathrm{IU} / \mathrm{L}$ ) to a prediction model resulted in significant gain in net reclassification improvement and integrated discrimination improvement of T2D prediction (all $p<0.001$ ).

Conclusions: Higher levels of GGT and ALT are associated with increased T2D risk. GGT $\geq 23 \mathrm{IU} / \mathrm{L}$ and ALT $\geq 21 \mathrm{IU} / \mathrm{L}$ may identify people at higher risk of developing T2D in this Chinese population.

\section{INTRODUCTION}

Liver, a vital organ in metabolism, plays an important role in maintaining glucose homeostasis. ${ }^{1}$ Markers of liver injury, such as alanine aminotransferase (ALT) and $\gamma$-glutamyltransferase (GGT), have been shown to be good surrogate measures of non-alcoholic fatty liver disease (NAFLD), the most common chronic liver conditions characterized by excess deposition of fat in

\section{Key messages}

- Increased levels of $\gamma$-glutamyltransferase (GGT) and alanine aminotransferase (ALT), even within the normal range, are associated with increased risk of incident type 2 diabetes independent of other diabetes risk factors in this Chinese population.

- GGT and ALT are useful markers for identifying people at higher risk of $\mathrm{T} 2 \mathrm{D}$, and the best cut-offs were 23 and $21 \mathrm{IU} / \mathrm{L}$ in this Chinese population.

- The other liver enzymes (aspartate aminotransferase, alkaline phosphatase, and lactate dehydrogenase) were not significantly associated with diabetes risk in the Chinese adults.

the liver, and associated with hepatic insulin resistance $^{2}$ and type 2 diabetes (T2D) risk. ${ }^{3}$ ALT, found predominately in the liver, has been considered the most specific marker for liver injury, ${ }^{4}$ while GGT is present on the surface of most cell types and highly active in liver, kidney, and pancreas. ${ }^{5}$ GGT is responsible for catabolism of extracellular glutathione and may be linked to oxidative stress ${ }^{6}$ and chronic inflammation, ${ }^{7}$ which are also important pathways for T2D development. Thus, ALT and GGT may be underlying biological markers linking between liver disease and T2D. Previous studies on ALT/GGT and T2D risk were conducted in Western ${ }^{2}$ 8-15 and Asian populations such as Japanese, ${ }^{16-18}$ South Koreans, ${ }^{19}{ }^{20}$ Thai, ${ }^{21}$ and Iranians, ${ }^{22}$ but not in a Chinese population.

The measurement of GGT and ALT involves well-standardized, simple, inexpensive, and routine tests with no requirement for fasting prior to venipuncture ${ }^{23}$ therefore, it is of clinical interest to explore whether these convenient biomarkers can help identify people at higher risk of developing T2D. However, existing studies found inconsistent findings: some studies showed that GGT and/or ALT improved T2D prediction significantly, ${ }^{16}$ 24-27 while others did not. ${ }^{22} 28-30$ Additionally, a recent study in a white and African-American 
population found that ALT $\geq 26 \mathrm{IU} / \mathrm{L}$ increased diabetes prediction substantially; ${ }^{27}$ while a Japanese study has identified a much lower cut-off value for ALT (13 IU/ L) ${ }^{16}$ Since Asians develop T2D at lower body mass index (BMI) compared to western populations, the observed difference indicates that liver may play a more important role in T2D development in relatively lean Asian populations. However, evidence is limited to confirm this assumption. Moreover, to the best of our knowledge, no study has examined the association of GGT, ALT with T2D risk, and their potential cut-off levels for T2D prediction in a Chinese population yet.

To address these issues, we conducted a nested casecontrol study within the Singapore Chinese Health Study (SCHS) to examine the relationship between liver enzymes and incident T2D risk in Chinese adults. Moreover, we evaluated the predictive utility of liver enzymes and identified appropriate cut-off values for T2D prediction.

\section{RESEARCH AND METHODS \\ Study population}

The design of SCHS was described previously. ${ }^{31}$ Briefly, SCHS was established between 1993 and 1998, and recruited 63257 Chinese adults aged 45-74 years. At recruitment, an in-person interview was conducted using a structured questionnaire to collect health-related information. Follow-up I (1999-2004) was conducted via telephone to update selected lifestyle habit and medical history. We recontacted 52322 participants successfully, and among them, 32535 participants donated their biospecimens during the follow-up I visit. Follow-up II (2006-2010) was conducted via telephone, and 39528 participants were recontacted successfully. The study protocol was approved by the Institutional Review Boards at the National University of Singapore and the University of Pittsburgh.

\section{Ascertainment of diabetes}

History of physician-diagnosed T2D risk was asked at baseline and both follow-ups by the question: "Have you been told by a doctor that you have diabetes?" If the answer was 'yes', participants were also asked for the age at which they were first diagnosed. The robustness and accuracy of the self-reported diabetes data was confirmed in a validation study: among 1651 cohort participants who reported prior diagnosis of diabetes either at baseline or at follow-up I interview, 98.9\% were confirmed by medical records (hospital-based discharge summary database) or a supplementary questionnaire about symptoms, diagnostic tests, and hypoglycemic therapy during a telephone interview. Some participants $(n=619)$ with self-reported diabetes refused or were not available for the validation study; however, their main characteristics (age, sex, BMI, etc) were similar with those in the validation study. ${ }^{32}$

\section{Case and control selection}

For the current analysis, we established a case-control study nested within SCHS of 571 cases and 571 matched controls. Cases and controls were free of physiciandiagnosed diabetes, cardiovascular disease, and cancer at baseline interview and blood collection (1999-2004). Cases were those who reported to be diagnosed with T2D during follow-up II (2006-2010). Controls were selected from the remaining participants who were free of T2D at follow-up II, and were matched for age ( \pm 3 years), date ( \pm 6 months) of blood collection, sex, and dialect group with cases on a 1:1 ratio. Moreover, the selected controls were screened for the presence of undiagnosed T2D at the time of blood donation by measuring hemoglobin Alc (HbAlc) level. Controls with $\mathrm{HbAlc} \geq 42 \mathrm{mmol} / \mathrm{mol}(6.0 \%)$ were ineligible for the study and excluded, and a replacement control with the same matching criteria was randomly chosen among the remaining eligible controls.

\section{Laboratory procedures}

Twenty milliliters of peripheral blood were collected from consenting participants, and transported to the laboratory immediately. All specimens were separated into plasma, serum, red blood cells, and buffy coat, and stored in $-80^{\circ}$ $\mathrm{C}$ freezers. For the current study, one aliquot of frozen plasma for each of study subjects were retrieved from the bioreposity. The plasma samples of matched case-control pairs were randomly placed next to each other with the case/control status blinded to the laboratory personnel, and processed and tested in the same batch. Plasma concentrations of ALT, aspartate aminotransferase (AST), alkaline phosphatase (ALP), lactate dehydrogenase (LDH), total cholesterol (TC), triglycerides (TG), highdensity lipoprotein cholesterol (HDL-C), and highsensitivity $\mathrm{C}$ reactive protein (CRP) were measured via colorimetric method on a chemistry analyzer (AU5800 Analyzer, Beckman Coulter, Brea, California, USA). Adiponectin levels were measured by ELISA (Bio-Rad Laboratories, Hercules, California, USA). HbAlc levels were measured by HPLC method using Bio-Rad Variant II System (Bio-Rad Laboratories) in red blood cells. All biochemical measurements were conducted at the National University Hospital Reference Laboratory.

Among 571 case-control pairs, although 279 cases had baseline HbAlc $\geq 48 \mathrm{mmol} / \mathrm{mol}(6.5 \%)$ and may be considered to have T2D according to current diagnostic criteria $^{33}$ they were not considered to have T2D because this was not used as criterion for diagnosis during the period of blood collection (1999-2004) and follow-up (2006-2010). Serum GGT was measured in another project focusing on cases with baseline HbA1c $<48 \mathrm{mmol} / \mathrm{mol}(6.5 \%)$ among 255 case-controls pairs with available serum samples (37 cases or controls had insufficient serum samples for the measurement). GGT was measured via the same colorimetric method mentioned above. For all liver enzymes, the within-assay 
coefficients of variation were $<8 \%$ and the between-assay coefficients of variation were all below $12 \%$.

\section{Statistical analysis}

ALT, AST, ALP, LDH, and GGT were divided into tertiles according to the distribution in the control samples and the lowest tertile served as the reference group. We used conditional logistic regression to model their associations with T2D adjusting for age (continuous), smoking status (never, past, and current smoker), alcohol intake (never, weekly, or daily), weekly moderate-to-vigorous activity levels $(<0.5,0.5-3.9$, and $\geq 4.0$ hours/week), education levels (no, primary school, secondary or above), history of hypertension (yes, no), fasting status (yes, no), and BMI (continuous). TG, HDL-C, CRP, and adiponectin levels (tertiles) were further adjusted in a separate model. We repeated the analyses in 292 case-control pairs (GGT: 255 pairs) with baseline HbA1c < $48 \mathrm{mmol} /$ mol $(6.5 \%)$. Moreover, we tested potential interactions with sex, BMI categories ( $<23 \mathrm{vs} \geq 23 \mathrm{~kg} / \mathrm{m}^{2}$ ), or fasting status. In the stratified analysis by BMI or fasting status, we used unconditional logistic regression models with additional adjustment for sex and dialect group.

Since GGT and ALT were associated with T2D, we further classified participants according to their GGT and ALT levels for T2D risk stratification. Receiver-operating characteristic (ROC) analysis and Youden index were used to derive the optimal cut-off values. ${ }^{34}$ To assess the predictive utility of dichotomized GGT and ALT, we established a parsimonious logistic regression model, including education level, physical activity, history of hypertension, BMI, TG, and HDL-C, using a forward selection procedure $(\mathrm{p}<0.05)$. The improvement in discrimination was examined by comparing area under ROC curve (AUC) between the parsimonious model and the model plus GGT and/or ALT using DeLong's method. ${ }^{35}$ Moreover, due to the limitation of AUC such as its insensitivity to model improvement, ${ }^{36}$ we also used the category-free net reclassification improvement (NRI) and integrated discrimination improvement (IDI) statistics recommended by Pencina et ll $^{3738}$ Furthermore, the goodness of fit of all models was assessed by Akaike information criteria (AIC), where lower AICs indicate better model fit. Analyses were performed with Stata software, V.11.0 (Stata Corp, College Station, Texas, USA). Two-sided $\mathrm{p}$ values $<0.05$ were considered to be statistically significant.

\section{RESULTS}

Among T2D cases, the mean age of diagnosis (SD) was 63.2 (6.4) years and the mean duration (SD) between blood donation and diagnosis of T2D was 4.0 (1.7) years. The baseline characteristics of cases and controls are shown in table 1. Cases had higher BMI, were more likely to have history of hypertension, and had higher levels of HbA1c, CRP, TG, GGT, ALT, AST, and ALP, and lower levels of adiponectin and HDL-C. No significant differences were found for education level, smoking, alcohol consumption, physical activity levels, and LDH levels.

ALT, AST, and GGT were moderately inter-related (correlation coefficients ranging from 0.50 to $0.67, \mathrm{p}<0.001$ ), while their correlations with ALP and LDH were weaker (correlation coefficients ranging from 0.08 to $0.28, p$ ranges from $<0.001$ to 0.21 ) (see online supplementary table S1). The associations between liver enzymes and T2D are presented in table 2 . In the whole data set (571 case-control pairs), increased ALT was associated with higher T2D risk (OR comparing extreme tertiles 2.33; $95 \%$ CI 1.52 to 3.55 ) in the final model. However, AST, ALP, and LDH showed no significant association. Moreover, the ALT-T2D association remained similar among 292 case-control pairs with $\mathrm{HbA1c}<48 \mathrm{mmol} /$ mol $(6.5 \%)$ (OR comparing highest vs lowest tertiles 2.38; $95 \%$ CI 1.21 to 4.66$)$. Higher GGT levels were also positively associated with increased T2D risk among 255 pairs with $\mathrm{HbAlc}<48 \mathrm{mmol} / \mathrm{mol}(6.5 \%)$ (OR comparing highest versus lowest tertiles 2.00; 95\% CI 1.01 to 3.96). Furthermore, no significant interactions were found with sex (see online supplementary table S2), BMI (see online supplementary table S3), and fasting status (see online supplementary table S4).

In this study, the best cut-off predictive values for T2D were $23 \mathrm{IU} / \mathrm{L}$ for GGT and $21 \mathrm{IU} / \mathrm{L}$ for ALT using Youden index in the ROC analysis. The sensitivities and specificities of the cut-off points were $72 \%$ and $48 \%$ for GGT, and $74 \%$ and $52 \%$ for ALT, respectively. A total of $61.6 \%$ participants had GGT $\geq 23 \mathrm{IU} / \mathrm{L}$ and $61.2 \%$ had ALT $\geq 21 \mathrm{IU} / \mathrm{L}$. GGT $\geq 23 \mathrm{IU} / \mathrm{L}$ was associated with a $69 \%$ greater odds of developing T2D (OR 1.69 (95\% CI 1.05 to 2.72)), while ALT $\geq 21 \mathrm{IU} / \mathrm{L}$ was associated with an $88 \%$ increased odds of developing T2D (OR 1.88 (95\% CI 1.41 to 2.52$)$ ). Additionally, compared to those who had GGT $<23$ IU $/ \mathrm{L}$ and ALT $<21 \mathrm{IU} / \mathrm{L}$, individuals with GGT $\geq 23 \mathrm{IU} / \mathrm{L}$ and ALT $\geq 21 \mathrm{IU} / \mathrm{L}$ were associated with a 2.5-fold odds of developing T2D (OR 2.47 (95\% CI 1.27 to 4.79)) (figure 1 and see online supplementary table S5).

The predictive performance of GGT and ALT is presented in table 3 and online supplementary table S6. Adding GGT (<23 vs $\geq 23 \mathrm{IU} / \mathrm{L})$ improved AUC from 0.76 in the base model to $0.77(\mathrm{p}=0.29)$. Comparatively, adding ALT (<21 vs $\geq 21 \mathrm{IU} / \mathrm{L})$ improved AUC from 0.74 to $0.75 \quad(p=0.02)$. Although the improvements in AUCs were minor, addition of GGT or ALT as binary variables to a prediction model resulted in significant gain in the category-free NRI and IDI of T2D prediction (all $\mathrm{p}<0.001$ ): $43.5 \%$ and $48.9 \%$ of T2D cases would be correctly predicted for higher T2D risk after inclusion of GGT or ALT, respectively. Additionally, inclusion of GGT or ALT showed better model fit with decreased AIC values compared to the base model (table 3).

\section{DISCUSSION}

In this case-control study nested within the SCHS, higher levels of GGT and ALT were positively associated 
Table 1 Baseline characteristics and liver enzymes of diabetes cases and matched controls, the SCHS

\begin{tabular}{|c|c|c|c|}
\hline & Cases $(n=571)$ & Controls $(n=571)$ & p Value* \\
\hline Age (years) at blood taken & $59.6 \pm 6.13$ & $59.7 \pm 6.22$ & - \\
\hline Gender (female) & $335(58.7)$ & $335(58.7)$ & - \\
\hline \multicolumn{4}{|l|}{ Dialect $(\%)$} \\
\hline Cantonese & $287(50.3)$ & $287(50.3)$ & \\
\hline Hokkien & $284(49.7)$ & $284(49.7)$ & \\
\hline BMI $\left(\mathrm{kg} / \mathrm{m}^{2}\right)$ & $24.8 \pm 3.62$ & $22.8 \pm 3.28$ & $<0.001$ \\
\hline Level of education (\%) & & & 0.66 \\
\hline No formal education & $104(18.2)$ & 99 (17.3) & \\
\hline Primary school & $255(44.7)$ & $233(40.8)$ & \\
\hline Secondary and above & $212(37.1)$ & $239(41.9)$ & \\
\hline History of hypertension (\%) & $265(46.4)$ & 148 (25.9) & $<0.001$ \\
\hline Cigarette smoking (\%) & & & 0.23 \\
\hline Never smokers & $410(71.8)$ & $425(74.4)$ & \\
\hline Former smokers & $63(11.0)$ & $71(12.4)$ & \\
\hline Current smokers & $98(17.2)$ & $75(13.1)$ & \\
\hline Weekly moderate-to-vigorous activity (\%) & & & 0.89 \\
\hline$<0.5$ hours/week & 456 (79.9) & $454(79.5)$ & \\
\hline 0.5-3.9 hours/week & $82(14.4)$ & $68(11.9)$ & \\
\hline$\geq 4$ hours/week & $33(5.8)$ & 49 (8.6) & \\
\hline Alcohol intake (\%) & & & 0.93 \\
\hline Abstainers & $498(87.2)$ & $497(87.0)$ & \\
\hline Weekly drinkers & $55(9.6)$ & $59(10.3)$ & \\
\hline Daily drinkers & $18(3.2)$ & $15(2.6)$ & \\
\hline Fasting status (yes) & $178(31.2)$ & $156(27.3)$ & 0.15 \\
\hline GGT† (IU/L) & $30(21-46)$ & $23(17-35)$ & $<0.001$ \\
\hline ALT (IU/L) & 27 (20-37) & $20(15-27)$ & $<0.001$ \\
\hline AST (IU/L) & $26(21-32)$ & $24(21-29)$ & $<0.001$ \\
\hline ALP (IU/L) & $84(68-101)$ & 77 (66-92) & $<0.001$ \\
\hline $\mathrm{LDH}(\mathrm{IU} / \mathrm{L})$ & $400 \pm 73$ & $403 \pm 77$ & 0.94 \\
\hline $\mathrm{TC}(\mathrm{mmol} / \mathrm{L})$ & $5.31 \pm 0.95$ & $5.20 \pm 0.85$ & 0.05 \\
\hline HDL-C (mmol/L) & $1.08 \pm 0.24$ & $1.23 \pm 0.32$ & $<0.001$ \\
\hline $\mathrm{TG}(\mathrm{mmol} / \mathrm{L})$ & $2.15(1.45-2.97)$ & $1.54(1.07-2.23)$ & $<0.001$ \\
\hline Adiponectin $(\mu \mathrm{g} / \mathrm{mL})$ & $7.00 \pm 2.70$ & $9.07 \pm 3.76$ & $<0.001$ \\
\hline C-reactive protein (mg/L) & $1.8(1.0-3.5)$ & $1.2(0.6-2.3)$ & $<0.001$ \\
\hline $\mathrm{HbA} 1 \mathrm{c}(\mathrm{mmol} / \mathrm{mol})$ & 51 & 38 & $<0.001$ \\
\hline $\mathrm{HbA1c}(\%)$ & $6.83 \pm 1.44$ & $5.55 \pm 0.27$ & $<0.001$ \\
\hline
\end{tabular}

Data are expressed as mean \pm SD for continuous variables (normally distributed) and median (IQR) for continuous variables (skewed distributed), and $n$ (percentage) for categorical variables.

Cases and controls are matched on age ( \pm 3 years), date of blood draw ( \pm 6 months), gender, and dialect group (Cantonese, Hokkien).

* $p$ values based on the McNemar's $\chi^{2}$ test for categorical variables, paired Student's t-test for normally distributed continuous variables, and Wilcoxon signed-rank test for skewed continuous variables.

†GGT concentrations were measured in 510 participants (255 case-control pairs with cases having baseline $\mathrm{HbA} 1 \mathrm{c}<48 \mathrm{mmol} / \mathrm{mol}(6.5 \%)$ ). ALP, alkaline phosphatase; ALT, alanine aminotransferase; AST, aspartate aminotransferase; BMI, body mass index; GGT, $\gamma$-glutamyl transferase; HbA1c, hemoglobin A1c; HDL-C, high-density lipoprotein cholesterol; LDH, lactate dehydrogenase; SCHS, Singapore Chinese Health Study; TC, total cholesterol; TG, triglycerides.

with the incident T2D risk independent of established T2D risk factors, including BMI, blood lipids, CRP, and adiponectin. Additionally, the best cut-off values for GGT and ALT were 23 and $21 \mathrm{IU} / \mathrm{L}$ in this Chinese population, and dichotomized GGT and ALT significantly improved T2D risk prediction.

Our finding of positive relations of GGT and ALT with incident T2D was largely consistent with previous studies across populations: a meta-analysis of 24 prospective studies reported a pooled relative risk of 1.34 (95\% CI 1.27 to 1.42 ) comparing highest versus lowest tertiles of GGT levels, ${ }^{39}$ and 1.66 (95\% CI 1.31 to $2.09 ; 17$ studies) for $\mathrm{ALT}^{23}$ although some included studies with relatively small sample sizes, ranging from 36 to 208 for T2D cases, did not observe the associations. A recent Mendelian randomization study further provided causal evidence for the association between GGT and insulin resistance. ${ }^{40}$ In the present study, we did not observe associations between AST and ALP with T2D, which was consistent with previous studies, ${ }^{1415212341}$ and this may be due to their lack of specificity for liver diseases. Additionally, no association was observed between LDH and T2D risk in the current study. Although increased LDH expression has affected glucose metabolism in a mechanistic study, ${ }^{42}$ few prospective human studies have assessed the LDH-T2D association. 
Table 2 ORs (95\% Cls) of T2D associated with different levels of liver enzymes, the SCHS

\begin{tabular}{|c|c|c|c|c|}
\hline \multirow[b]{2}{*}{ Variables } & \multicolumn{3}{|c|}{ Tertiles of liver enzymes } & \multirow[b]{2}{*}{ p for trend $\mathbf{d}^{*}$} \\
\hline & T1 & T2 & T3 & \\
\hline \multicolumn{5}{|l|}{ Whole dataset } \\
\hline \multicolumn{5}{|l|}{ ALT } \\
\hline Median (range) & $14(6-17)$ & $21(18-24)$ & $33(25-130)$ & \\
\hline Cases/controls & $93 / 218$ & $152 / 163$ & $326 / 190$ & \\
\hline Model 1 & 1.00 & $1.98(1.34-2.92)$ & $3.73(2.56-5.44)$ & $<0.001$ \\
\hline Model 2 & 1.00 & $1.56(1.02-2.40)$ & $2.33(1.52-3.55)$ & $<0.001$ \\
\hline \multicolumn{5}{|l|}{ AST } \\
\hline Median (range) & $20(12-22)$ & $25(23-27)$ & $33(28-135)$ & \\
\hline Cases/controls & $173 / 212$ & $153 / 181$ & $245 / 178$ & \\
\hline Model 1 & 1.00 & $0.96(0.69-1.33)$ & $1.39(1.01-1.91)$ & 0.03 \\
\hline Model 2 & 1.00 & $0.90(0.63-1.30)$ & $1.10(0.77-1.58)$ & 0.52 \\
\hline \multicolumn{5}{|l|}{ ALP } \\
\hline Median (range) & $61(23-70)$ & $78(71-86)$ & $101(87-401)$ & \\
\hline Cases/controls & $155 / 191$ & $168 / 194$ & $248 / 186$ & \\
\hline Model 1 & 1.00 & $1.14(0.82-1.58)$ & $1.78(1.28-2.48)$ & $<0.001$ \\
\hline Model 2 & 1.00 & $1.08(0.74-1.56)$ & $1.39(0.96-2.02)$ & 0.07 \\
\hline \multicolumn{5}{|l|}{ LDH } \\
\hline Median (range) & $331(216-366)$ & $394(367-426)$ & $473(427-713)$ & \\
\hline Cases/controls & $205 / 191$ & $191 / 191$ & $175 / 189$ & \\
\hline Model 1 & 1.00 & $0.85(0.60-1.20)$ & $0.71(0.50-1.01)$ & 0.06 \\
\hline Model 2 & 1.00 & $0.77(0.52-1.13)$ & $0.72(0.49-1.07)$ & 0.12 \\
\hline \multicolumn{5}{|c|}{ Limited to cases with baseline $\mathrm{HbA} 1 \mathrm{c}<48 \mathrm{mmol} / \mathrm{mol}(6.5 \%)$ and their matched controls } \\
\hline \multicolumn{5}{|l|}{ GGT† } \\
\hline Median (range) & $15(10-18)$ & $24(19-28)$ & $43(29-741)$ & \\
\hline Cases/controls & $48 / 92$ & $70 / 80$ & $137 / 83$ & \\
\hline Model 1 & 1.00 & $1.53(0.81-2.87)$ & $2.91(1.61-5.26)$ & $<0.001$ \\
\hline Model 2 & 1.00 & $1.28(0.61-2.68)$ & $2.00(1.01-3.96)$ & 0.03 \\
\hline \multicolumn{5}{|l|}{ ALT } \\
\hline Median (range) & $14(6-16)$ & $20(17-23)$ & $33(24-119)$ & \\
\hline Cases/controls & $45 / 98$ & $85 / 98$ & $162 / 96$ & \\
\hline Model 1 & 1.00 & $2.08(1.15-3.74)$ & $3.85(2.15-6.90)$ & $<0.001$ \\
\hline Model 2 & 1.00 & $1.84(0.94-3.59)$ & $2.38(1.21-4.66)$ & 0.05 \\
\hline \multicolumn{5}{|l|}{ AST } \\
\hline Median (range) & $20(13-22)$ & $25(23-27)$ & $33(28-105)$ & \\
\hline Cases/controls & $93 / 104$ & $82 / 104$ & $117 / 84$ & \\
\hline Model 1 & 1.00 & $0.84(0.51-1.37)$ & $1.38(0.86-1.37)$ & 0.12 \\
\hline Model 2 & 1.00 & $0.75(0.43-1.30)$ & $1.03(0.60-1.77)$ & 0.80 \\
\hline \multicolumn{5}{|l|}{ ALP } \\
\hline Median (range) & $60(28-70)$ & $78(71-86)$ & $101(87-172)$ & \\
\hline Cases/controls & $90 / 101$ & $90 / 94$ & $112 / 97$ & \\
\hline Model 1 & 1.00 & $1.01(0.65-1.59)$ & $1.33(0.84-2.10)$ & 0.21 \\
\hline Model 2 & 1.00 & $0.94(0.56-1.57)$ & $0.92(0.53-1.59)$ & 0.76 \\
\hline \multicolumn{5}{|l|}{ LDH } \\
\hline Median (range) & $330(216-363)$ & $392(364-425)$ & $472(427-687)$ & \\
\hline Cases/controls & $107 / 98$ & $107 / 98$ & $78 / 96$ & \\
\hline Model 1 & 1.00 & $1.03(0.62-1.70)$ & $0.67(0.62-1.70)$ & 0.06 \\
\hline Model 2 & 1.00 & $0.94(0.52-1.68)$ & $0.68(0.37-1.24)$ & 0.13 \\
\hline
\end{tabular}

ORs of liver enzymes in the whole dataset and among cases with baseline $\mathrm{HbA} 1 \mathrm{c}<48 \mathrm{mmol} / \mathrm{mol}(6.5 \%)$ and their matched controls were computed using conditional logistic regression models with adjustment for following covariates.

Model 1: adjusted for age at blood taken (continuous), smoking (never, past, and current smoker), alcohol intake (never, weekly, or daily), weekly moderate-to-vigorous activity $(<0.5,0.5-3$, and $\geq 4$ hours/week), education level (no, primary school, secondary or above), history of hypertension (yes, no), fasting status (yes, no), and BMI (continuous).

Model 2: model 1 plus adjusted triglycerides ( $\mathrm{mmol} / \mathrm{L})$, high-density lipoprotein cholesterol (mmol/L), C-reactive protein $(\mathrm{mg} / \mathrm{L})$, and adiponectin $(\mu \mathrm{g} / \mathrm{mL})$ (all in tertiles).

*Linear trend was tested using the median level of each tertile of liver enzymes.

†GGT concentrations were measured in 510 participants (255 case-control pairs with cases having baseline $\mathrm{HbA1c}<48 \mathrm{mmol} / \mathrm{mol}(6.5 \%)$ ). ALP, alkaline phosphatase; ALT, alanine aminotransferase; AST, aspartate aminotransferase; BMI, body mass index; GGT, $\gamma$-glutamyl transferase; HbA1c, hemoglobin A1c; LDH, lactate dehydrogenase; SCHS, Singapore Chinese Health Study; T2D, type 2 diabetes. 


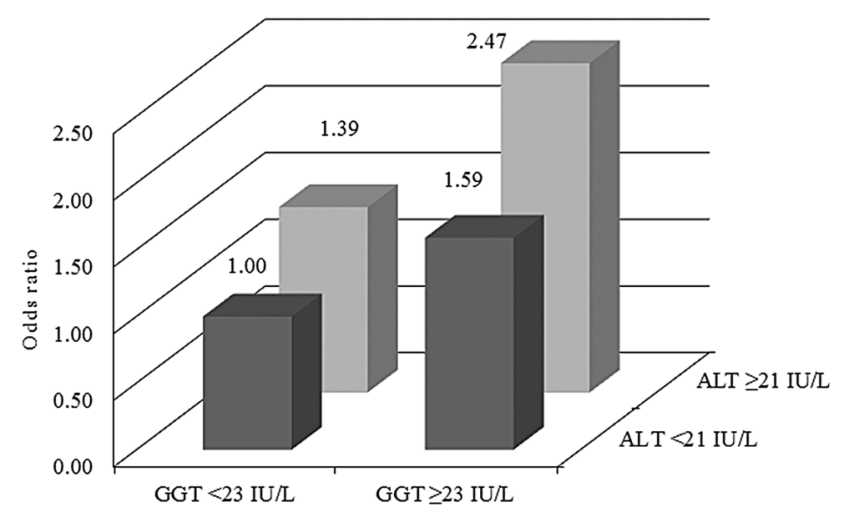

Figure 1 Total T2D risk according to join distribution of GGT and ALT. ALT concentrations were measured in 1142 participants (571 case-control pairs). GGT concentrations were measured in 510 participants ( 255 case-control pairs with cases having baseline $\mathrm{HbA} 1 \mathrm{c}<48 \mathrm{mmol} / \mathrm{mol}(6.5 \%))$. Multivariable model adjusted for age at blood taken (continuous), smoking (never, past, and current smoker), alcohol intake (never, weekly, or daily), weekly moderate-to-vigorous activity $(<0.5,0.5-3$, and $\geq 4$ hours/ week), education level (no, primary school, secondary or above), history of hypertension (yes, no), fasting status (yes, no), BMI (continuous), plasma tertile concentrations of triglycerides, high-density lipoprotein cholesterol, C-reactive protein, and adiponectin. ALT, alanine aminotransferase; BMI, body mass index; GGT, $\gamma$-glutamyl transferase; T2D, type 2 diabetes.

Elevated GGT and ALT levels were linked to T2D development as useful surrogate measures of NAFLD characterized by hepatic fat accumulation. ${ }^{43}$ NAFLD may indicate fat deposition in other organs such as skeletal muscle, the myocardium, and the pancreas, which predispose individuals to T2D risk. ${ }^{43}$ However, a Korean study showed positive associations between GGT/ALT and T2D risk among participants without fatty liver, and suggested that alternative pathways existed. ${ }^{20}$
Additionally, ALT was linked to T2D via hepatic insulin resistance, ${ }^{14}$ and GGT through oxidative stress ${ }^{6}$ and inflammation. ${ }^{7}$ However, adjustment for CRP (low-grade inflammation marker) had no influence on the association in our study and in other studies, ${ }^{2} 131520$ suggesting that GGT may be involved in the pathogenesis of T2D through other mechanisms. Moreover, previous evidence showed that the relations of GGT and ALT with T2D were also independent of other important pathologies in T2D development such as whole-body insulin resistance $^{2} 14$ and blood lipids, ${ }^{2} 131619$ and the current study further showed that the associations were also independent of adiponectin.

Moreover, our study found that GGT and ALT levels improved T2D risk prediction, and this was supported by several previous studies. ${ }^{16}$ 24-27 GGT, ALT, glucose, HbAlc, HDL-C, and TG collectively improved the discriminatory power (indicated by AUC) above the basic model in EPIC-Potsdam study. ${ }^{24}$ GGT, together with BMI, waist circumference, and $\mathrm{TG}$, showed reasonable discrimination (measured by c-statistics) in D.E.S.I.R study. ${ }^{25}$ Moreover, inclusion of HbAlc and GGT in a simple clinical model showed significant improvement in T2D prediction (AUC, NRI, and IDI) in British older men and women. ${ }^{26}$ Furthermore, a recent study found that $\mathrm{ALT} \geq 26 \mathrm{IU} / \mathrm{L}$ improved T2D prediction (AUC, NRI, and IDI) among white and African-Americans living in the USA. ${ }^{27}$ Additionally, a Japanese study has found that GGT $(\geq 18 \mathrm{IU} / \mathrm{L})$ or ALT $(\geq 13 \mathrm{IU} / \mathrm{L})$ had similar discriminatory power with BMI for predicting T2D. ${ }^{16}$ We have identified a lower ALT cut-off value $(21 \mathrm{IU} / \mathrm{L})$ for $\mathrm{T} 2 \mathrm{D}$ prediction compared to the US study, although higher than the Japanese study. As for GGT, some studies observed little improvement in T2D prediction (indicated by NRI) when including GGT and other novel biomarkers to the basic model. ${ }^{28-30}$ In the current study, including GGT in the model significantly

Table 3 Summary statistics to assess binary liver enzymes in predicting incident T2D

\begin{tabular}{|c|c|c|c|c|c|}
\hline \multirow[t]{2}{*}{ Variable } & \multirow[t]{2}{*}{ Best cut-offs } & \multicolumn{4}{|l|}{ Multivariable model } \\
\hline & & Discrimination (AUC (95\% CI)) & Calibration (AIC) & NRI & IDI \\
\hline Base model & & $0.74(0.71$ to 0.77$)$ & 1394 & & \\
\hline ALT & 21 & $0.75(0.73 \text { to } 0.78)^{*}$ & 1370 & 0.53 & 0.02 \\
\hline Base model & & $0.76(0.72$ to 0.80$)$ & 613 & & \\
\hline GGT & 23 & $0.77(0.73$ to 0.81$) \dagger$ & 609 & 0.41 & 0.01 \\
\hline Base model & & $0.76(0.72$ to 0.80$)$ & 613 & & \\
\hline $\mathrm{ALT}+\mathrm{GGT}$ & - & $0.77(0.73$ to 0.81$) \dagger$ & 609 & - & - \\
\hline
\end{tabular}

Base model included education level (no, primary school, secondary and above), weekly moderate-to-vigorous activity $(<0.5,0.5-3.9$, and $\geq 4$ hours/week), history of hypertension (yes, no), plasma concentrations of triglycerides ( $\mathrm{mmol} / \mathrm{L}$ ) (tertiles) and high-density lipoprotein cholesterol (mmol/L) (tertiles), and BMI (continuous).

Multivariable model adjusted for all the variables included in the base model.

${ }^{*}$ ALT concentrations were measured in 1142 participants (571 case-control pairs). Compared with the base model, the increment in AUC value was statistically significant $(p<0.05)$.

TGGT concentrations were measured in 510 participants (255 case-control pairs with cases having baseline $\mathrm{HbA} 1 \mathrm{c}<48 \mathrm{mmol} / \mathrm{mol}(6.5 \%)$ ). Compared with the base model (AUC, $0.76(0.72-0.80)$ ), increment in AUC value by adding GGT to the base model was not significant $(p=0.36)$. Additionally, increment in AUC value by adding GGT together with ALT to the base model was not significant either ( $p=0.20)$. AIC, Akaike information criterion; ALT, alanine aminotransferase; AUC, area under ROC curve; BMI, body mass index; GGT, $\gamma$-glutamyl transferase; IDI, integrated discrimination improvement; NRI, net reclassification improvement; T2D, type 2 diabetes. 
improved NRI, but not AUC, and the cut-off value $(\geq 23 \mathrm{IU} / \mathrm{L})$ is slightly higher than the one identified in a Japanese study. ${ }^{16}$

The strength of the current study included adjustment for well-established diabetes risk factors (including BMI, CRP, lipids, and adiponectin), and using comprehensive statistical methods (AUC, NRI, and IDI) to explore the predictive utility of liver enzymes. However, there are some limitations. First, we measured liver enzymes only once and may not represent long-term profile. Previous studies have shown that the within-person coefficient of variation of GGT ranged between $12.2 \%$ and $13.8 \%$ in repeated measures up to 1 year. ${ }^{44}{ }^{45}$ In addition, the impact of single measurement of GGT in the current study would most likely lead to non-differential misclassification and underestimate the GGT-T2D association. For example, a previous study in the D.E.S.I.R. cohort found that correction for the within-person variation of GGT slightly strengthened the association between GGT and T2D risk. ${ }^{46}$ Second, incident diabetes was obtained from self-reported information, thus undiagnosed diabetes may exist. However, we have measured HbAlc, which was updated as a diagnosis criterion of diabetes in 2010 by the American Diabetes Association. ${ }^{33}$ Similar associations were found when analyzing the data in the total samples or in those cases with HbAlc $\leq 48 \mathrm{mmol} /$ mol $(6.5 \%)$ at baseline. Third, although we did not observe any interaction with ALT or GGT, our sample size may be small and thus underpowered to detect the interaction. Fourth, we did not measure hepatitis B and C infection, which could result in elevated liver enzymes. Moreover, we did not have data on insulin resistance; however, the association remained in previous studies adjusting for direct measurement of insulin resistance. ${ }^{2}{ }^{14}$ Last but not least, HbA1c was a selection criterion for controls in the current study; therefore, we could not include it in our prediction model. A study from the Netherlands showed that liver function tests led to small but significant improvement in T2D prediction above basic model; however, the improvement disappeared when HbAlc was included in the basic model. ${ }^{29}$

\section{CONCLUSION}

Increased levels of GGT and ALT are associated with increased risks of incident T2D in this Chinese population. Additionally, GGT and ALT are useful markers for identifying people at higher risk of T2D, and the best cut-offs were 23 and $21 \mathrm{IU} / \mathrm{L}$ in this Chinese population. Further researches are needed to validate the findings, particularly the predictive utility of liver enzymes for T2D, and investigate the biological mechanisms for the associations.

\footnotetext{
Author affiliations

${ }^{1}$ Saw Swee Hock School of Public Health, National University of Singapore and National University Health System, Singapore, Singapore

${ }^{2}$ Duke-NUS Graduate Medical School Singapore, Singapore, Singapore
}

${ }^{3}$ Division of Cancer Control and Population Sciences, University of Pittsburgh Cancer Institute, Pittsburgh, Pennsylvania, USA

${ }^{4}$ Department of Epidemiology, Graduate School of Public Health, University of Pittsburgh, Pittsburgh, Pennsylvania, USA

${ }^{5}$ Department of Epidemiology and Statistics, School of Public Health, Tongji Medical College, Huazhong University of Science and Technology, Wuhan, Hubei Province, China

Acknowledgements The authors thank Siew-Hong Low for supervising the fieldwork of the SCHS, Renwei Wang for the maintenance of the cohort database, and Mimi C Yu for founding the cohort study.

Contributors Y-LW analyzed the data and wrote the first draft, and all authors contributed to the interpretation of the results and critical revision of the manuscript for important intellectual content and approved the final version of the manuscript. All authors had access to the data.

Funding This work was supported by the National Medical Research Council, Singapore (NMRC/CIRG/1354/2013) and National Institutes of Health, USA (R01 CA144034 and UM1 CA182876).

\section{Competing interests None declared.}

Ethics approval The study protocol was approved by the Institutional Review Boards at the National University of Singapore and the University of Pittsburgh.

Provenance and peer review Not commissioned; externally peer reviewed.

Data sharing statement Data are from the Singapore Chinese Health Study, and any data sharing request should be directed to the principal investigators at yuanj@upmc.edu (J-MY) and woonpuay.koh@duke-nus.edu.sg (W-PK).

Open Access This is an Open Access article distributed in accordance with the Creative Commons Attribution Non Commercial (CC BY-NC 4.0) license, which permits others to distribute, remix, adapt, build upon this work noncommercially, and license their derivative works on different terms, provided the original work is properly cited and the use is non-commercial. See: http:// creativecommons.org/licenses/by-nc/4.0/

\section{REFERENCES}

1. Gavin N, Levinthal AST. Liver disease and diabetes mellitus. Clin Diabetes 1999;17.

2. Hanley AJ, Williams K, Festa A, et al. Elevations in markers of liver injury and risk of type 2 diabetes: the insulin resistance atherosclerosis study. Diabetes 2004:53:2623-32.

3. Ballestri S, Zona S, Targher G, et al. Nonalcoholic fatty liver disease is associated with an almost twofold increased risk of incident type 2 diabetes and metabolic syndrome. Evidence from a systematic review and meta-analysis. J Gastroenterol Hepatol 2016;31:936-44.

4. Giannini EG, Testa R, Savarino V. Liver enzyme alteration: a guide for clinicians. CMAJ 2005;172:367-79.

5. Hanigan MH, Frierson HF Jr. Immunohistochemical detection of gamma-glutamyl transpeptidase in normal human tissue. $J$ Histochem Cytochem 1996;44:1101-8.

6. Turgut $\mathrm{O}$, Tandogan I. Gamma-glutamyltransferase to determine cardiovascular risk: shifting the paradigm forward. J Atheroscler Thromb 2011;18:177-81.

7. Lee DH, Jacobs DR Jr. Association between serum gamma-glutamyltransferase and $\mathrm{C}$-reactive protein. Atherosclerosis 2005; 178:327-30.

8. Andre P, Balkau B, Born C, et al. Hepatic markers and development of type 2 diabetes in middle aged men and women: a three-year follow-up study. The D.E.S.I.R. Study (Data from an Epidemiological Study on the Insulin Resistance syndrome). Diabetes Metab 2005;31:542-50.

9. Ford ES, Schulze MB, Bergmann MM, et al. Liver enzymes and incident diabetes: findings from the European Prospective Investigation into Cancer and Nutrition (EPIC)-Potsdam Study. Diabetes Care 2008;31:1138-43.

10. Fraser A, Harris R, Sattar N, et al. Alanine aminotransferase, gamma-glutamyltransferase, and incident diabetes: the British Women's Heart and Health Study and meta-analysis. Diabetes Care 2009;32:741-50.

11. Goessling W, Massaro JM, Vasan RS, et al. Aminotransferase levels and 20-year risk of metabolic syndrome, diabetes, and cardiovascular disease. Gastroenterology 2008;135:1935-44, 44.e1. 
12. Monami M, Bardini G, Lamanna C, et al. Liver enzymes and risk of diabetes and cardiovascular disease: results of the Firenze Bagno a Ripoli (FIBAR) study. Metab Clin Exp 2008;57:387-92.

13. Sattar N, Scherbakova O, Ford I, et al. Elevated alanine aminotransferase predicts new-onset type 2 diabetes independently of classical risk factors, metabolic syndrome, and C-reactive protein in the west of Scotland coronary prevention study. Diabetes 2004;53:2855-60.

14. Vozarova B, Stefan N, Lindsay RS, et al. High alanine aminotransferase is associated with decreased hepatic insulin sensitivity and predicts the development of type 2 diabetes. Diabetes 2002;51:1889-95.

15. Wannamethee SG, Shaper AG, Lennon L, et al. Hepatic enzymes, the metabolic syndrome, and the risk of type 2 diabetes in older men. Diabetes Care 2005;28:2913-18.

16. Doi Y, Kubo M, Yonemoto K, et al. Liver enzymes as a predictor for incident diabetes in a Japanese population: the Hisayama study. Obesity (Silver Spring) 2007;15:1841-50.

17. Nakanishi N, Suzuki K, Tatara K. Serum gamma-glutamyltransferase and risk of metabolic syndrome and type 2 diabetes in middle-aged Japanese men. Diabetes Care 2004;27:1427-32.

18. Sato KK, Hayashi T, Nakamura Y, et al. Liver enzymes compared with alcohol consumption in predicting the risk of type 2 diabetes: the Kansai Healthcare Study. Diabetes Care 2008;31:1230-6.

19. Cho $\mathrm{NH}$, Jang $\mathrm{HC}$, Choi $\mathrm{SH}$, et al. Abnormal liver function test predicts type 2 diabetes: a community-based prospective study. Diabetes Care 2007;30:2566-8.

20. Kim CH, Park JY, Lee KU, et al. Association of serum gamma-glutamyltransferase and alanine aminotransferase activities with risk of type 2 diabetes mellitus independent of fatty liver. Diabetes Metab Res Rev 2009;25:64-9.

21. Jiamjarasrangsi W, Sangwatanaroj S, Lohsoonthorn V, et al. Increased alanine aminotransferase level and future risk of type 2 diabetes and impaired fasting glucose among the employees in a university hospital in Thailand. Diabetes Metab 2008;34:283-9.

22. Tohidi M, Harati $\mathrm{H}$, Hadaegh $\mathrm{F}$, et al. Association of liver enzymes with incident type 2 diabetes: a nested case control study in an Iranian population. BMC Endocr Disord 2008;8:5

23. Kunutsor SK, Apekey TA, Walley J. Liver aminotransferases and risk of incident type 2 diabetes: a systematic review and meta-analysis. Am J Epidemiol 2013;178:159-71.

24. Schulze MB, Weikert $C$, Pischon $T$, et al. Use of multiple metabolic and genetic markers to improve the prediction of type 2 diabetes: the EPIC-Potsdam Study. Diabetes Care 2009;32:2116-19.

25. Balkau B, Lange C, Vol S, et al. Nine-year incident diabetes is predicted by fatty liver indices: the French D.E.S.I.R. study. BMC Gastroenterol 2010;10:56.

26. Wannamethee SG, Papacosta O, Whincup PH, et al. The potential for a two-stage diabetes risk algorithm combining non-laboratory-based scores with subsequent routine non-fasting blood tests: results from prospective studies in older men and women. Diabet Med 2011;28:23-30.

27. Lorenzo C, Hanley AJ, Rewers MJ, et al. Discriminatory value of alanine aminotransferase for diabetes prediction: the Insulin Resistance Atherosclerosis Study. Diabet Med 2016;33:348-55.
28. Abbasi A, Bakker SJ, Corpeleijn E, et al. Liver function tests and risk prediction of incident type 2 diabetes: evaluation in two independent cohorts. PLOS ONE 2012;7:e51496.

29. Raynor LA, Pankow JS, Duncan BB, et al. Novel risk factors and the prediction of type 2 diabetes in the Atherosclerosis Risk in Communities (ARIC) study. Diabetes Care 2013;36:70-6.

30. Marques-Vidal P, Schmid R, Bochud M, et al. Adipocytokines, hepatic and inflammatory biomarkers and incidence of type 2 diabetes. The CoLaus study. PLoS ONE 2012;7:e51768.

31. Hankin JH, Stram DO, Arakawa K, et al. Singapore Chinese Health Study: development, validation, and calibration of the quantitative food frequency questionnaire. Nutr Cancer 2001;39:187-95.

32. Odegaard AO, Pereira MA, Koh WP, et al. Coffee, tea, and incident type 2 diabetes: the Singapore Chinese Health Study. Am J Clin Nutr 2008;88:979-85

33. American Diabetes Association. Standards of medical care in diabetes-2016. Diabetes Care 2016;39(Suppl 1):S1-106.

34. Youden WJ. Index for rating diagnostic tests. Cancer 1950;3:32-5.

35. DeLong ER, DeLong DM, Clarke-Pearson DL. Comparing the areas under two or more correlated receiver operating characteristic curves: a nonparametric approach. Biometrics 1988;44:837-45.

36. Pepe MS, Janes $\mathrm{H}$, Longton $\mathrm{G}$, et al. Limitations of the odds ratio in gauging the performance of a diagnostic, prognostic, or screening marker. Am J Epidemiol 2004;159:882-90.

37. Pencina MJ, D'Agostino RB Sr, Steyerberg EW. Extensions of net reclassification improvement calculations to measure usefulness of new biomarkers. Stat Med 2011;30:11-21.

38. Pencina MJ, D'Agostino RB Sr, D'Agostino RB Jr, et al. Evaluating the added predictive ability of a new marker: from area under the ROC curve to reclassification and beyond. Stat Med 2008;27:157-72; discussion 207-12.

39. Kunutsor SK, Abbasi A, Adler Al. Gamma-glutamyl transferase and risk of type II diabetes: an updated systematic review and dose-response meta-analysis. Ann Epidemiol 2014;24:809-16.

40. Conen D, Vollenweider P, Rousson V, et al. Use of a Mendelian randomization approach to assess the causal relation of gamma-glutamyltransferase with blood pressure and serum insulin levels. Am J Epidemiol 2010;172:1431-41.

41. Nannipieri M, Gonzales C, Baldi S, et al. Liver enzymes, the metabolic syndrome, and incident diabetes: the Mexico City diabetes study. Diabetes Care 2005;28:1757-62.

42. Ainscow EK, Zhao $C$, Rutter GA. Acute overexpression of lactate dehydrogenase-A perturbs beta-cell mitochondrial metabolism and insulin secretion. Diabetes 2000;49:1149-55.

43. Unger RH. Minireview: weapons of lean body mass destruction: the role of ectopic lipids in the metabolic syndrome. Endocrinology 2003;144:5159-65

44. Lazo M, Selvin E, Clark JM. Brief communication: clinical implications of short-term variability in liver function test results. Ann Intern Med 2008;148:348-52.

45. Kunutsor SK, Khan H, Laukkanen JA. $\gamma$-Glutamyltransferase and risk of sudden cardiac death in middle-aged Finnish men: a new prospective cohort study. J Am Heart Assoc 2016;5:e002858.

46. André P, Balkau B, Born C, et al. Three-year increase of gamma-glutamyltransferase level and development of type 2 diabetes in middle-aged men and women: the D.E.S.I.R. cohort. Diabetologia 2006;49:2599-603. 